\title{
Phlegmasia cerulea dolens: before and after lysis
}

\author{
Sean Daniel Kalagher · Derek Douglas Kane
}

Received: 15 April 2014/ Accepted: 9 May 2014/Published online: 25 May 2014

(C) SIMI 2014

A 77-year-old woman, immobile due to advanced rheumatoid arthritis, presented to the emergency department with a 3-day course of a progressively painful and purple left lower extremity (Fig. 1a). On examination, the limb was discolored, swollen, warmer than the right leg, and very tender. The distal pulses were entirely absent, and only a faint left femoral pulse was palpable. Phlegmasia cerulea dolens (from Latin for inflamed, blue, and painful) was the clinical impression [1] and confirmed with Doppler ultrasound, which demonstrated an occlusive thrombus in the left femoral vein. Sensation and motor function of the limb remained intact. She was started on a heparin drip, and brought to interventional radiology for endovascular therapy. Fig. 1b shows a venogram of the left iliofemoral segment with extensive occlusive deep venous thrombus (DVT) that extended to the left popliteal vein (not shown).

Catheter directed lysis was performed overnight resulting in Dopplerable distal pulses and improved symptoms; however, interval venography (not shown) revealed significant residual thrombus burden. The digital subtraction venogram of the left iliofemoral segment in Fig. 1c depicts improved patency and flow following subsequent endovascular mechanical thrombectomy. Fig. 1d shows the improvement in the patient's left leg $12 \mathrm{~h}$ later $(36 \mathrm{~h}$ after presentation). Her pain resolved, and her distal pulses

\section{S. D. Kalagher $(\varangle)$}

Division of Interventional Radiology, UND School of Medicine,

St. Alexius Medical Center, 900 East Broadway, Bismarck, ND 58501, USA

e-mail: skalagher@yahoo.com

\section{D. Kane}

Department of General Surgery, UND School of Medicine, Mid Dakota Clinic, 401 North 9th Street, Bismarck, ND 58501, USA e-mail: dkane@primecare.org became palpable. She was discharged on warfarin. Although prolonged immobility was the presumptive risk factor for her, consultation with a hematologist/oncologist
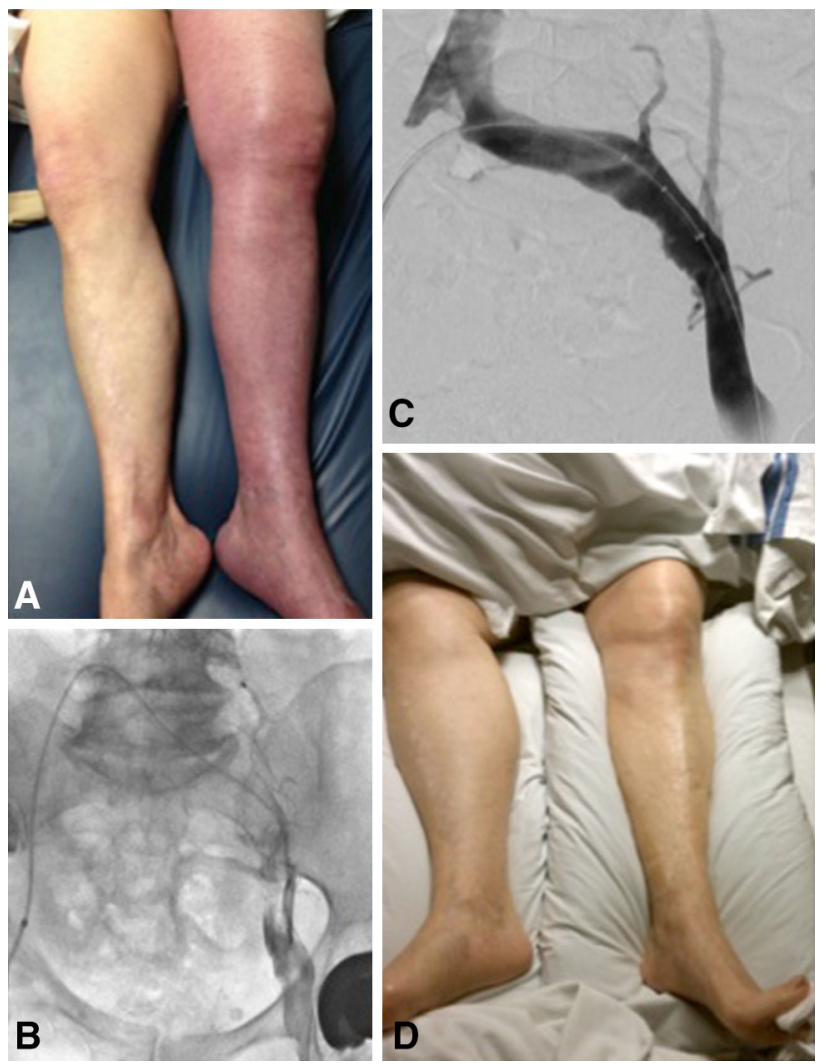

Fig. 1 a Left lower limb at presentation. b Venogram of the left iliofemoral segment with extensive occlusive DVT that extended to the left popliteal vein (not shown). c Digital subtraction venogram following endovascular treatment depicts improved patency and flow. d Left lower limb at $36 \mathrm{~h}$ 
Table 1 Typical clinical factors distinguishing venous versus arterial obstruction in acute limb ischemia

\begin{tabular}{llllll}
\hline Occlusion & Temperature & Swelling & Color & Extent & Cap. Refill \\
\hline Venous & Warmer & Significant & Purple/blue & Often entire limb & Fast \\
Arterial & Cooler & None & Pale or mottled & Usually distal (foot or leg) & Delayed (>5 s) \\
\hline
\end{tabular}

was initiated to exclude underlying malignancy and clotting disorder.

Phlegmasia cerulea dolens (PCD) represents the most severe acute consequence of DVT. Prompt diagnosis along with pharmacological and endovascular therapy is the key to avoiding complications such as gangrene (40-60\%), amputation (20-50\%), and death (25-40\%) [2]. However, reperfusion by any means should be deferred when extensive irreversible tissue damage is present as reperfusion injury can result in multi-organ failure and death [3].

A discolored pulseless leg due to venous occlusion PCD should be distinguished from arterial occlusion on clinical grounds, and confirmed with imaging (Table 1). The extremity in PCD is purplish, warmer than the unaffected limb, swollen, and often demonstrates faster than normal capillary refill. In contradistinction, the extremity of arterial occlusion is typically pale or mottled, cooler than the unaffected limb, not swollen, and capillary refill will be delayed [4]. Also, it is much less common for the skin discoloration to involve the entire lower extremity in arterial occlusion while this is often the case in PCD.
Duplex ultrasound can identify or exclude occlusion in both the arteries and veins of the lower extremity, can characterize flow dynamics, and serves as a good first choice for imaging. CT and MRI imaging are useful, but slow flow can mimic thrombus on these modalities confounding interpretation. Catheter venography and arteriography remain the gold standard, and can be therapeutic.

Conflict of interest None.

\section{References}

1. Gregoire R (1938) La Phlebite bleue (phlegmasia cerulea dolens). Press Med 46(71):1313-1315

2. Chinsakchai K, Duis K, Moll FL, de Borst GJ (2011) Trends in management of phlegmasia cerulea dolens. Vasc Endovascular Surg 45(1):5-14

3. Lessne ML, Bajwa J, Hong K (2012) Fatal reperfusion injury after thrombolysis for phlegmasia cerulea dolens. J Vasc Interv Radiol 23(5):681-686

4. Creager MA, Kaufman JA, Conte MS (2012) Acute limb ischemia. N Engl J Med 366(23):2198-2206 